\title{
Optical control of excited-state lifetimes
}

\author{
David Andrews and David S. Bradshaw
}

Electronic excitation of particles in fluorescent materials can now be controlled using laser-assisted energy-transfer techniques.

When light is absorbed by a fluorescent nanoparticle, the resulting excited state has a decay lifetime that is primarily determined by two intrinsic optical routes. One is decay through emission of fluorescence, while the other is by resonance-energy transfer (RET), the transfer of the excitation to another site. It has recently become evident that the passage of a moderately intense, off-resonant laser beam may enable optical control of excited-state lifetimes in such a system. The general formula for the excited-state lifetime, $\tau$, is very simply expressible as $\tau^{-1}=\tau_{\mathrm{FL}}^{-1}+\tau_{\mathrm{RET}}^{-1}+K I$. The first two terms on the right are well known. They correspond to excited-state lifetimes associated only with fluorescence and RET, respectively. The additional, newly discovered term exhibits a proportionality to the intensity of an off-resonant laser beam, $I$. Detailed equations for the contributions to the proportionality constant, $K$, have been reported in several recent publications. Using the theory of quantum electrodynamics, we have shown that $K$ expresses a dependence on laser-controlled fluorescence and laser-assisted RET.

Suppose we have a single molecule or nanoparticle (such as a quantum dot) without any other in its immediate vicinity. The input of an off-resonant laser beam may modify the intensity of the molecule's fluorescent emission. ${ }^{1,2}$ The all-optical mechanism involves no net absorption or stimulated light emission, since the throughput beam undergoes forward scattering and emerges unchanged (see Figure 1). Specifically, elastic forward scattering of the off-resonant light engages by nonlinear coupling with the decay process. In essence, the probe laser confers optical nonlinearity on the fluorescent emission (see Figure 2). Unlike the well-known enhancement of emission by coupling with surface plasmons, ${ }^{3,4}$ this mechanism signifies that such modifications to spontaneous fluorescent emission may be produced without the presence of any surface, through direct interaction with the oscillating electric field of the throughput electromagnetic radiation.

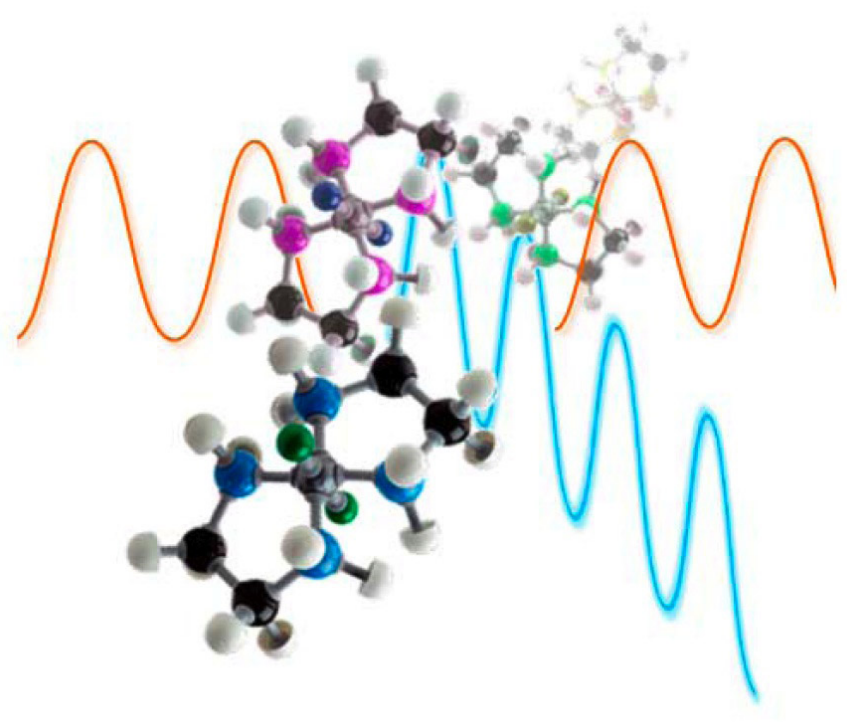

Figure 1. Representation of laser-controlled fluorescence. Blue line: Fluorescence. Orange: Off-resonant throughput laser beam.

We have determined that fluorescence lifetimes, under specified conditions, can be reduced by $\sim 10 \%$ for an input laser irradiance of $10^{11} \mathrm{~W} / \mathrm{cm}^{2}$ and proportionately more for higher intensities. Even at a level of $10^{10} \mathrm{~W} / \mathrm{cm}^{2}$, we anticipate that the change in decay kinetics will be readily measurable with modulation-based instrumentation. Time-correlated photon-detection techniques might represent a methodology particularly well-suited to such measurements when deployed in an adaptation of the setup for standard laser-fluorescence spectroscopy. With a tunable laser source, the capacity to control the wavelength of the throughput radiation introduces an additional experimental variable, offering a second dimension to sample interrogation. Establishing the variation with laser-pulse intensity will also validate the mechanism at work.

We have also examined the ability of throughput light to exert control over RET, a process that is favored when an electronically excited component (acting as donor) relays its excitation to 


\section{Newsroom}

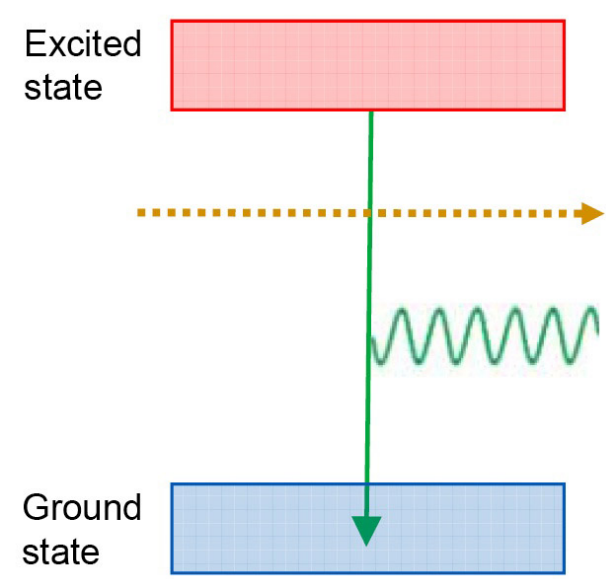

Figure 2. Energy-level diagram for laser-controlled fluorescence, a third-order nonlinear optical process. Electronic states (and their vibrational manifolds) are represented by boxes, the wavy line is emitted fluorescence, the horizontal dashed arrow denotes the off-resonant laser beam, and the green vertical arrow is an electronic transition.

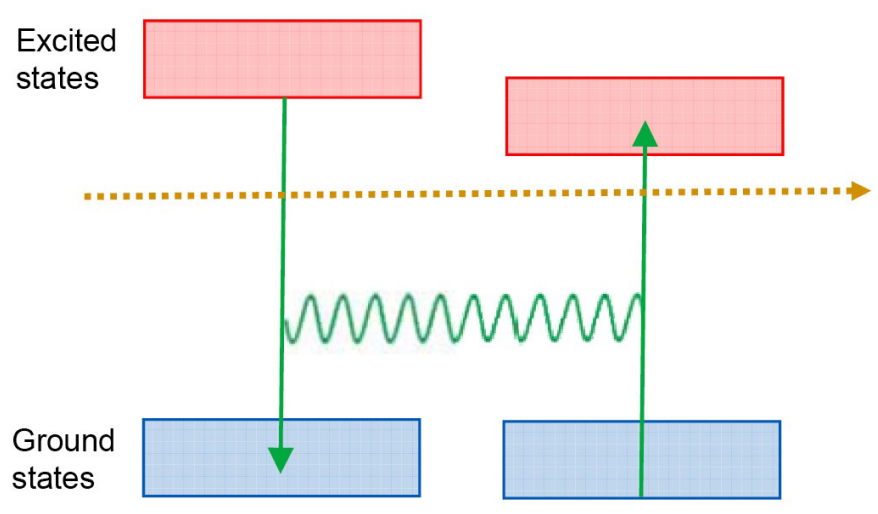

Figure 3. Energy-level scheme for laser-assisted resonance-energy transfer, a fourth-order process. As Figure 2, except that the wavy line now denotes energy transfer. The energy levels for an initially excited particle are on the left, those for a nearby neighbor on the right.

a neighboring particle with suitable acceptor levels. When RET occurs spontaneously, it usually represents the dominant process for the decay of the species that holds the initial excitation. ${ }^{5}$ On input of the off-resonant laser field, another, higher-order, all-optical mechanism may occur that again entails stimulated forward scattering of the input beam (see Figure 3). Our calculations show that laser-assisted RET may confer a several-fold decrease in the decay lifetime of a donor ${ }^{6}$ for moderately intense laser irradiances of around $10^{12} \mathrm{~W} / \mathrm{cm}^{2}$.

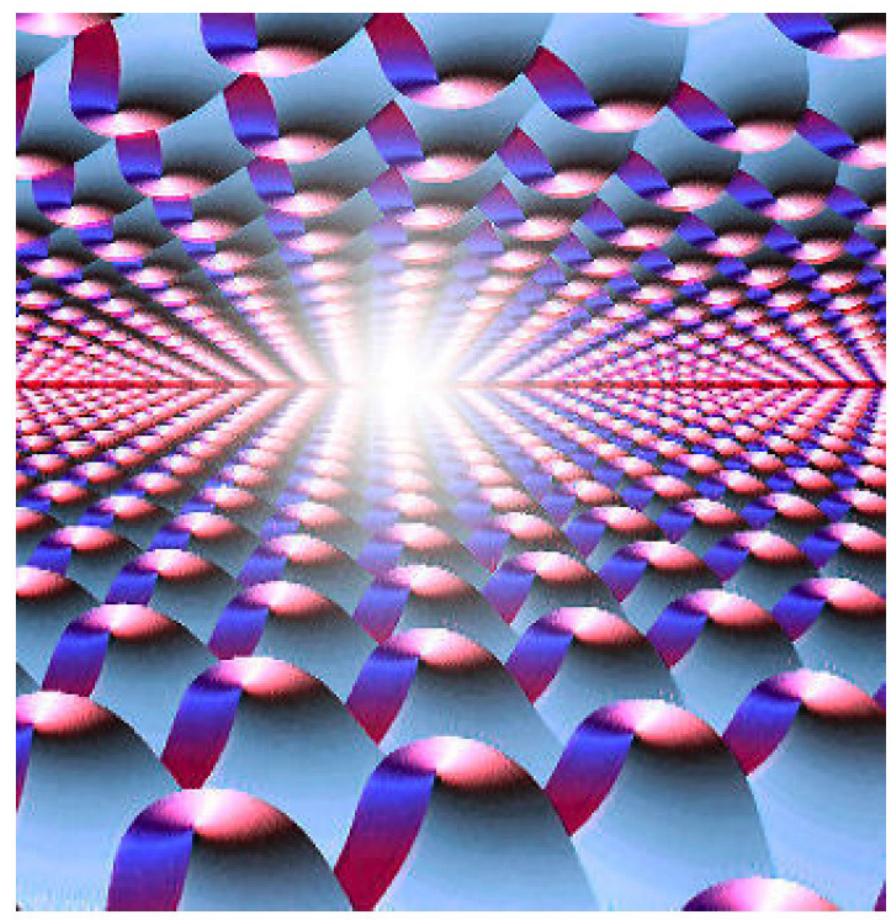

Figure 4. All-optical switching device comprising parallel 2D arrays, each composed of nanoparticles (spacer layer not shown).

We recently proposed an all-optical switching device based on this laser-assisted energy-transfer process. Working on the principle that conventional RET can be eliminated through a suitably designed geometric configuration (transition dipoles placed mutually orthogonally to each other and to the donoracceptor pair displacement), the transfer of energy will then occur only on application of the off-resonant laser. Switching action thus ensues, since donor decay by the transport of excitation energy is activated or deactivated when the throughput beam is 'on' or 'off,' respectively. ${ }^{7}$ To exploit this switching action, one can envisage a sandwich structure comprising a pair of parallel 2D arrays (each composed of nanoparticle pixels) on either side of an optically passive spacer layer (see Figure 4). Such a configuration provides a parallel signal-processing capability. ${ }^{8}$

Although the mechanisms for laser modifications to fluorescence and energy transfer involve different orders of optical response, they may both contribute to reducing excited-state lifetimes. Our attention now focuses on examination of their combined effect, which could lead to unanticipated levels of response. One reason for our interest in such a scenario is the 
possibility of an increased cycle frequency for excitation and decay, increasing signal response and deferring the onset of optical saturation. Progress in theory is revealing more detail about the characteristics of $K$ and its determining factors. For example, we have already identified dispersion properties that can be exploited by tuning the throughput light in pre-resonance regions. There are potentially associated spectroscopic applications, because the influence of a tunable probe beam offers the scope to secure 2D spectra from complex materials, where the fluorescence is monitored as a function of not only the pump but also the probe wavelength. With pulsed sources, new tiers of kinetic information should also become available. As always, the path from new discoveries in optics to applications seems likely to prove short and well-trodden.

Research on energy transfer and fluorescence in the quantumelectrodynamics group at the University of East Anglia is supported by the UK's Leverhulme Trust.

\section{Author Information}

\section{David Andrews and David S. Bradshaw}

School of Chemistry

University of East Anglia

Norwich, UK

David Andrews leads research on fundamental molecular photonics and energy transport, optomechanical forces, and nonlinear optics. He has published over 250 research papers and 10 books. His current research focuses on optical nanomanipulation and switching, and light harvesting in nanostructures.

David Bradshaw is a postdoctoral research associate whose current interests include resonance-energy transfer, all-optical switching, and optical binding. He has co-authored over 35 peer-reviewed papers, including an encyclopedia article on applied spectroscopy.
References

1. D. S. Bradshaw and D. L. Andrews, Mechanism for optical enhancement and suppression of fluorescence, J. Phys. Chem. A 113 (24), pp. 6537-6539, 2009. doi:10.1021/jp901830w

2. D. S. Bradshaw and D. L. Andrews, All-optical control of molecular fluorescence, Phys. Rev. A 81 (1), p. 013424, 2010. doi:10.1103/PhysRevA.81.013424

3. H. Rigneault, J. Capoulade, J. Dintinger, J. Wenger, N. Bonod, E. Popov, T. W. Ebbesen, and P.-F. Lenne, Enhancement of single-molecule fluorescence detection in subwavelength apertures, Phys. Rev. Lett. 95 (11), p. 117401, 2005 doi:10.1103/PhysRevLett.95.117401

4. P. Anger, P. Bharadwaj, and L. Novotny, Enhancement and quenching of single-molecule fluorescence, Phys. Rev. Lett. 96 (11), p. 113002, 2006. doi:10.1103/PhysRevLett.96.113002

5. D. L. Andrews and D. S. Bradshaw, in D. L. Andrews ed., Resonance energy transfer, Encyclopedia of Applied Spectroscopy, pp. 533-554, Wiley-VCH, Weinheim, 2009. ISBN: 978-3-527-40773-6.

6. P. Allcock, R. D. Jenkins, and D. L. Andrews, Laser-assisted resonance-energy transfer, Phys. Rev. A 61 (2), p. 023812, 2000. doi:10.1103/PhysRevA.61.023812

7. D. S. Bradshaw and D. L. Andrews, Optically controlled resonance energy transfer: mechanism and configuration for all-optical switching, J. Chem. Phys. 128 (14) p. 144506,2008 . doi:10.1063/1.2894319

8. D. S. Bradshaw and D. L. Andrews, All-optical switching between quantum dot nanoarrays, Superlatt. Microstruct. 47 (2), pp. 308-313, 2010 doi:10.1016/j.spmi.2009.10.017 